\title{
Treinamento muscular respiratório em pacientes em desmame da ventilação mecânica
}

\author{
Respiratory muscle training in patients weaning from mechanical ventilation
}

Fernanda dos Santos Pascotini ${ }^{1,2}$, Camila Denardi ${ }^{3}$, Graziana Oliveira Nunes ${ }^{1}$, Maria Elaine Trevisan²,4 Vívian da Pieve Antunes $^{2,3}$

'Departamento de Fisioterapia do Hospital de Caridade Dr. Astrogildo de Azevedo (HCAA) - Santa Maria (RS), Brasil.

${ }^{2}$ Curso de Distúrbios da Comunicação Humana da Universidade Federal de Santa Maria (UFSM) - Santa Maria (RS), Brasil.

${ }^{3}$ Centro Universitário Franciscano (UNIFRA) - Santa Maria (RS), Brasil.

${ }^{4}$ Departamento de Fisioterapia e Reabilitação da UFSM - Santa Maria (RS), Brasil.

DOI: http://dx.doi.org/10.7322/abcshs.v39i1.253

\section{RESUMO}

Introdução: A fraqueza da musculatura respiratória é uma das principais causas da dificuldade e/ou insucesso no desmame. Para minimizar os efeitos da ventilação mecânica (VM) prolongada, os fisioterapeutas utilizam o treinamento muscular respiratório, sendo o Threshold IMT ${ }^{\circledR}$ o método mais utilizado. Objetivo: Avaliar a eficácia do treinamento muscular respiratório com o uso do aparelho Threshold $I M T^{\circledR}$, sobre parâmetros respiratórios de pacientes em desmame da VM. Métodos: Os pacientes foram distribuídos aleatoriamente em Grupo Controle e Grupo Experimental (Gl e GII). Foram avaliados no primeiro dia do início do desmame quanto à força muscular respiratória: Pressão inspiratória máxima/Pressão Expiratória Máxima (PImáx/PEmáx), volume corrente (VC), frequência respiratória (FR) e cardíaca (FC). Diariamente, durante sete dias, o Gl recebeu três sessões de fisioterapia convencional e o Gll realizou, adicionalmente, treinamento muscular respiratório (TMR) com o Threshold IMT ${ }^{\circledR}$, uma vez ao dia, no período da tarde, conectado à traqueostomia, sendo três séries de dez repetições com carga de $20 \%$ da PImáx. Os dados foram tratados estatisticamente, adotando-se o nível de significância $\alpha=0,05$. Resultados: Observou-se aumento $(p=0,02)$ na FR e redução da PImáx $(p=0,04)$ no $G I$, demonstrando aumento do trabalho respiratório e perda de força muscular entre o primeiro e sétimo dia de desmame. No Gll, as variáveis não sofreram alterações significativas, observando-se a manutenção da função respiratória. Conclusão: Sendo assim, o TMR foi benéfico, garantindo a manutenção dos parâmetros respiratórios, podendo ser um aliado para o desmame.

Palavras-chave: desmame do respirador; capacitação; força muscular; músculos respiratórios.

\begin{abstract}
Introduction: The weakness of the respiratory muscles is a major cause of difficulty and/or failure of weaning. To minimize the effects of prolonged mechanical ventilation (MV), the physiotherapists use respiratory muscle training, being the Threshold IMT ${ }^{\circledR}$ the most commonly used method. Objective: To evaluate the effectiveness of respiratory muscle training using the Threshold IMT ${ }^{\circledR}$ device on respiratory parameters in patients weaning from MV. Methods: Patients were distributed randomly in the control group and the experimental group (GI and GII). On the first day of the weaning, the respiratory muscle strength (PImax/PEmax), tidal volume (TV), respiratory rate (RR) and heart rate (HR) were evaluated. For seven days, the Gl received three sessions of conventional physiotherapy and Gll has additionally held respiratory muscle training (RMT) with the Threshold IMT ${ }^{\circledR}$, once a day, in the afternoon, connected to tracheostomy, and three sets of ten repetitions, at a load of $20 \%$ of PImax. The data were statistically treated by adopting a significance level $\alpha=0.05$. Results: There was an increase $(p=0.02)$ in $R R$ and a decrease in the PImax $(p=0.04)$ in $\mathrm{Gl}$, demonstrating increased work of breathing and loss of muscle strength between the first and the seventh day of weaning. In Gll, the variables did not change significantly, observing the maintenance of respiratory function. Conclusion: Thus, RMT is beneficial, ensuring the maintenance of respiratory parameters, which can be an ally in weaning.
\end{abstract}

Keywords: ventilator weaning; training; muscle strength; respiratory muscles.

Recebido em: 17/07/2013

Revisado em: 15/10/2013

Aprovado em: 20/12/2013

Endereço para correspondência

Fernanda dos Santos Pascotini - Avenida Presidente Vargas, 2291 - Centro - CEP: 97015-513 - Santa Maria (RS), Brasil - E-mail: fepascotini@hotmail.com Conflito de interesses: nada a declarar. 


\section{INTRODUÇÃO}

Os pacientes críticos, dependentes da Ventilação Mecânica (VM) controlada por longos períodos (mais de 48 horas), apresentam importante comprometimento dos músculos respiratórios, que hipotrofiam e perdem força e resistência, devido à inatividade. A fraqueza da musculatura respiratória é uma das principais causas da dificuldade e/ou insucesso no desmame, pois impede que o paciente respire espontaneamente de forma adequada ${ }^{1-3}$. Segundo a Associação de Medicina Intensiva Brasileira (AMIB $)^{4}, 24 \%$ dos pacientes em VM apresentam falha no desmame.

Para minimizar os efeitos da VM prolongada, os fisioterapeutas utilizam o treinamento muscular respiratório (TMR), cuja função é habilitar músculos específicos a realizarem a função para qual são destinados, objetivando tanto força muscular quanto endurance. Sendo assim, é necessário que esses músculos apresentem mínimas condições fisiológicas, como a integridade da condução nervosa e circulação adequada ${ }^{5}$.

O treinamento muscular inspiratório é uma intervenção que vem sendo adotada para melhorar a força e a resistência à fadiga dos músculos inspiratórios em pacientes com alteração da função respiratória e cardíaca ${ }^{6}$. Pode ser realizado por meio de respiração contrarresistida, com dispositivos de carga alinear ou linear pressórica, sendo a carga linear por meio do dispositivo Threshold $I M T^{\oplus}$, o método mais utilizado para treinamento específico da musculatura inspiratória ${ }^{5}$.

O Threshold IMT ${ }^{\oplus}$ é um resistor inspiratório, por sistema de mola, com uma válvula unidirecional que abre durante a expiração, não havendo nenhuma resistência durante esta fase da respiração, e fecha na inspiração, promovendo resistência e, dessa forma, fortalecendo a musculatura inspiratória ${ }^{7}$. É utilizado para aumentar a força e endurance dos músculos respiratórios, melhorar as condições de trabalho, e reduzir a fadiga ${ }^{5}$, porém, a utilização deste dispositivo, com o propósito de facilitar o desmame da $\mathrm{VM}$, ainda não é consenso entre os estudos.

Com base nestas considerações, o objetivo do presente estudo foi avaliar a eficácia do TMR com o uso do aparelho Threshold IMT ${ }^{\oplus}$, sobre parâmetros respiratórios de pacientes em desmame da VM.

\section{MÉTODOS}

Pesquisa experimental do tipo pré e pós-intervenção, com análise quantitativa dos dados.

Foram incluídos no estudo, pacientes de ambos os gêneros, com idade igual ou superior a 40 anos, traqueostomizados, em processo de desmame da VM, independente da patologia de base. Foram excluídos os pacientes com doença neuromuscular ou que apresentassem desconforto respiratório durante o treinamento.

O estudo foi aprovado pelo Comitê de Ética em Pesquisa local, sob o número 194.2009.2, e o Termo de Consentimento Livre e
Esclarecido — de acordo com a resolução 196/96 do Conselho Nacional de Saúde - foi assinado pelo familiar responsável pelo paciente.

Preenchendo os critérios de inclusão, os pacientes foram distribuídos aleatoriamente em Grupo Controle e Grupo Experimental (GI e GII). Os pacientes de ambos os grupos foram avaliados no primeiro dia do início do desmame, utilizando-se uma ficha de avaliação incluindo dados de identificação, história da doença pregressa e atual, sinais vitais, tempo de VM, força muscular respiratória: Pressão Inspiratória Máxima/Pressão Expiratória Máxima (PImáx e PEmáx), volume corrente (VC), frequência respiratória (FR) e frequência cardíaca (FC).

Para realizar a mensuração da PImáx, o manovacuômetro analógico (Suporte R), com limite operacional de -300 a $+300 \mathrm{~cm} / \mathrm{H}_{2} \mathrm{O}$, foi conectado diretamente na cânula de traqueostomia, e foi solicitado ao paciente que expirasse todo o ar até o volume residual (VR) e, através do método de oclusão simples, foi solicitado ao paciente que inspirasse até a capacidade pulmonar total $(\mathrm{CPT})^{9,10}$. O tempo de oclusão de 40 segundos, sugerido por Black e Hyatt ${ }^{9}$, não foi tolerado pelos pacientes e, desta forma, foi realizado uma oclusão de 20 segundos.

Para mensurações de PEmáx, foi solicitado ao paciente inspiração até a CPT, o orifício do manovacuômetro foi ocluído por vinte segundos, e foi solicitado ao paciente que expirasse até o VR. Foram realizadas três manobras de PImáx e PEmáx, com descanso de 40 segundos entre cada uma delas e o paciente recebendo estímulo verbal da pesquisadora ${ }^{10,11}$. Foi considerado o maior valor obtido e, caso a diferença entre as duas maiores medidas ultrapassasse $10 \%$, uma nova mensuração foi realizada.

Nas situações em que o nível de consciência estava baixo, foi observado o ciclo respiratório, realizada a oclusão no início da inspiração, para aferir a PImáx, e no início da expiração, para aferir a PEmáx, utilizando comando verbal para inspirar ou expirar com o máximo esforço ${ }^{10}$.

O VC e a FR foram obtidos pelo Respiradyne II $^{\oplus}$ (Model 5-7930P Sher Wood Medical Co), conectado à traqueostomia durante um minuto, onde o paciente deveria respirar normalmente. A FC foi obtida pelo monitor cardíaco (marca Dixtal DX 2022).

Diariamente, durante sete dias, o Grupo Controle (GI) e o Grupo Experimental (GII) receberam três sessões de fisioterapia respiratória, composta por manobras de higiene brônquica e aspiração traqueal, e três sessões de fisioterapia motora, com o paciente em decúbito dorsal, composta por dez repetições dos seguintes exercícios: flexão/extensão de ombros, cotovelos, quadril e joelhos, abdução/adução de ombros e quadril, planti-flexão dos pés, além de alongamentos de membros inferiores.

O GII realizou, adicionalmente às três sessões de fisioterapia respiratória e motora, o TMR, uma vez ao dia, no período da tarde, sempre após higiene brônquica e aspiração traqueal. Para o TMR (GII), foi utilizado o aparelho Threshold IMT (Respironics), que oferece resistência inspiratória ajustável de -7 a $-41 \mathrm{~cm} / \mathrm{H}_{2} \mathrm{O}$, conectado à traqueostomia. $\mathrm{O}$ paciente foi posicionado em decúbito 
dorsal, cabeceira elevada a $45^{\circ}$, e foram solicitadas três séries de dez inspirações lentas e profundas, utilizando carga de treinamento de $20 \%$ da PImáx ${ }^{5}$, com intervalo para descanso de dois minutos entre cada série. Durante todo o tempo de treinamento, de aproximadamente 20 minutos, foi monitorada a pressão arterial sistêmica (PAS) e a saturação periférica de oxigênio $\left(\mathrm{SpO}_{2}\right)$ por meio do monitor cardíaco e, caso atingissem valores fora da normalidade, a terapia deveria ser interrompida.

O treinamento foi realizado por sete dias consecutivos, sendo que ambos os grupos foram reavaliados no dia seguinte ao término do treinamento, ou seja, no oitavo dia, no período da tarde.

Os dados foram analisados por estatística descritiva e a normalidade pelo teste de Shapiro-Wilk. Para a comparação intragrupos, foi utilizado o Teste $t$ de Student para amostras dependentes. Na comparação entre grupos, foi utilizado o teste de Mann-Whitney para as variáveis com distribuição não normal (PImáx, PEmáx), e Teste $t$ de Student para amostras independentes para as variáveis com distribuição normal (FC, FR, VC), ao nível de significância de $5 \%$. As análises foram realizadas por meio do programa estatístico SPSS 17.

\section{RESULTADOS}

A amostra foi constituída por 14 pacientes (três do gênero masculino e onze do gênero feminino), distribuídos aleatoriamente em Grupo Controle (GI) e Grupo Experimental (GII). A caracterização dos grupos está apresentada na Tabela 1 .

Os grupos apresentaram homogeneidade nas variáveis de caracterização idade, tempo de VM, e nas variáveis do estudo antes das intervenções (FC, FR, VC, PImáx e PEmáx).

Observou-se aumento $(\mathrm{p}=0,02)$ na $\mathrm{FR}$ e redução da PImáx $(\mathrm{p}=0,04)$ no GI, demonstrando aumento do trabalho respiratório e perda de força muscular entre o primeiro e sétimo dia de desmame (Figuras 1 e 2, respectivamente).

No GII, não foram observadas alterações significativas da FC, FR, PImáx, PEmáx e VC ( $>00,05)$, destacando a manutenção das variáveis neste grupo (Figuras 1, 2 e 3, respectivamente).

Tabela 1: Caracterização dos grupos de estudo

\begin{tabular}{|l|c|c|c|}
\hline Variáveis & \multicolumn{1}{|c|}{$\begin{array}{c}\text { GI } \\
\mathbf{n = 7}\end{array}$} & $\begin{array}{c}\text { Gll } \\
\mathbf{n}=\mathbf{7}\end{array}$ & Valor $\mathbf{p}$ \\
\hline $\begin{array}{l}\text { Sexo (M/F) } \\
\text { Idade (anos) }\end{array}$ & $3 / 4$ & $0 / 7$ & \\
\hline $\begin{array}{l}\text { Tempo de ventilação } \\
\text { mecânica (dias) }\end{array}$ & $72,40 \pm 11,90$ & $67,00 \pm 13,90$ & 0,45 \\
\hline $\begin{array}{l}\text { Patologias de base } \\
\text { TCE }\end{array}$ & $16,29 \pm 3,35$ & $17,14 \pm 7,42$ & 0,78 \\
\hline \multicolumn{1}{|c|}{ AVE } & 2 & 3 & - \\
\hline Falha no desmame & 5 & 4 & - \\
\hline
\end{tabular}

Gl: Grupo Controle; Gll: Grupo Experimental; M: masculino; F: feminino; TCE: traumatismo cranioencefálico; AVE: acidente vascular encefálico; *média \pm desvio padrão

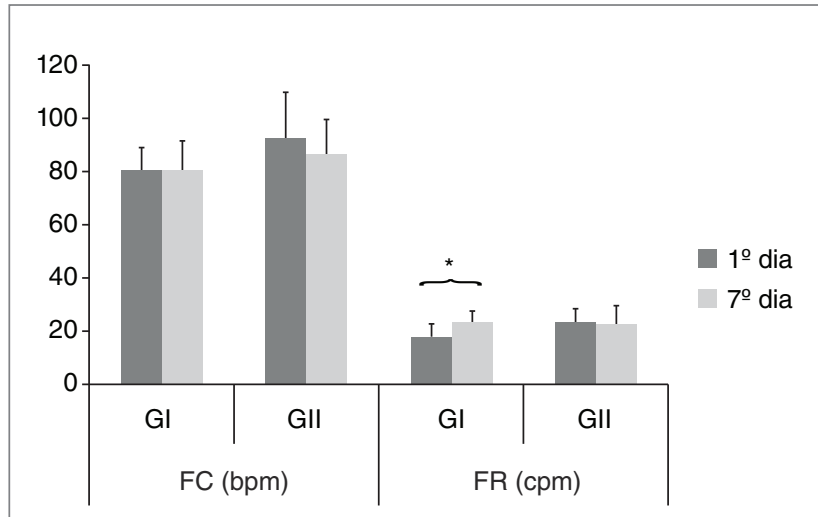

Gl: Grupo Controle; Gll: Grupo Experimental; FC: frequência cardíaca; FR: frequência respiratória; bpm: batimentos por minuto; cpm: ciclos por minuto

Figura 1: Média e desvio padrão das variáveis frequência cardíaca e respiratória no primeiro e sétimo dia de desmame em ambos os grupos. Nível de significância - * $p<0,05$, Teste $t$ de Student

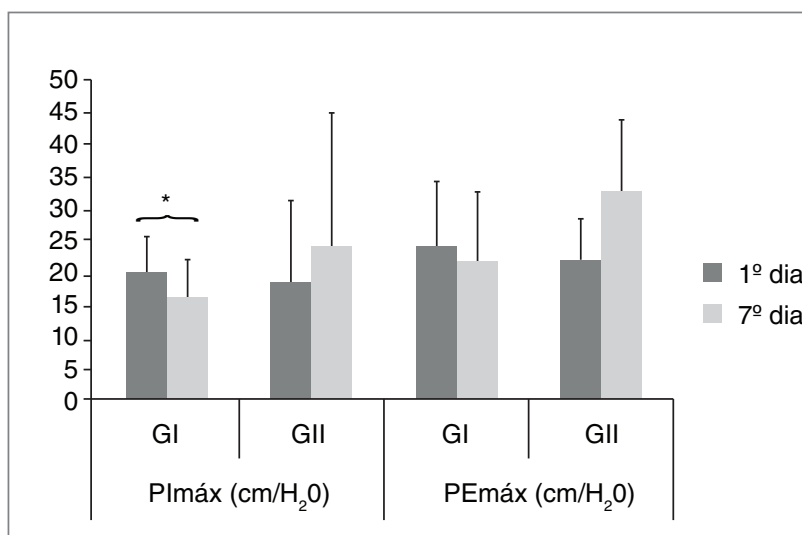

Gl: Grupo Controle; GII: Grupo Experimental; PImáx: pressão inspiratória máxima; PEmáx: pressão expiratória máxima

Figura 2: Média e desvio padrão das variáveis pressão inspiratória e expiratória máxima no primeiro e sétimo dias de desmame em ambos os grupos. Nível de significância - ${ }^{*} p<0,05$, Teste $t$ de Student

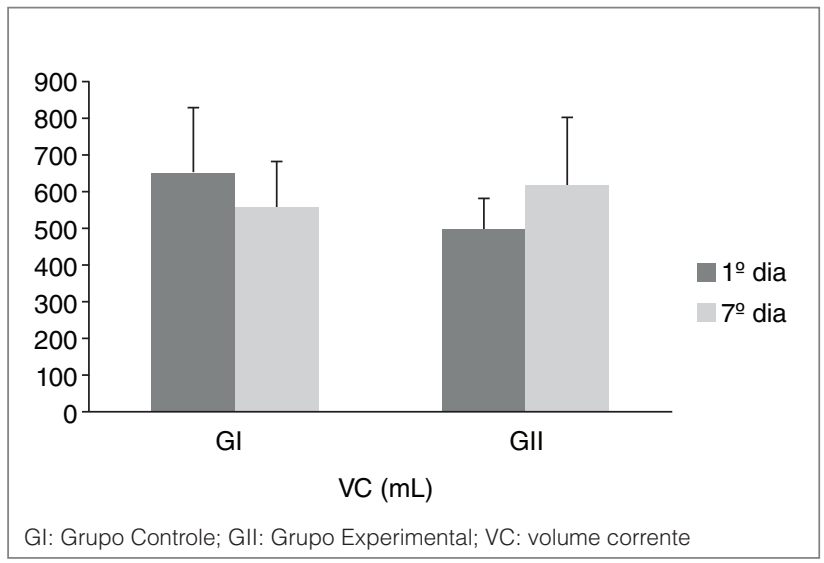

Figura 3: Média e desvio padrão da variável volume corrente no primeiro e sétimo dias de desmame em ambos os grupos 


\section{DISCUSSÃO}

O tempo médio de VM observado neste estudo foi de $16,71 \pm 5,55$ dias. A literatura aponta que o tempo de ventilação artificial, a partir do qual se iniciam os efeitos deletérios nos músculos respiratórios, varia de 48 a 72 horas ${ }^{12,13}$. Um dos principais prejuízos causados em decorrência do uso do ventilador mecânico é a fraqueza muscular respiratória ${ }^{1,2}$, que hipotrofia a musculatura devido ao desuso, já que o ventilador substitui a bomba ventilatória, deixando os músculos inativos.

Neste estudo, observou-se que os pacientes que foram submetidos ao TMR (GII), mantiveram os valores de PImáx. Esta manutenção na força inspiratória provavelmente contribuiu para o desmame, tendo em vista que nenhum dos participantes do GII teve falha no desmame do ventilador mecânico. Estes resultados favoráveis não foram evidenciados no grupo que não realizou treinamento muscular inspiratório (GI), no qual a PImáx decresceu e a falha no desmame ocorreu em três pacientes.

Vários estudos prévios constataram melhora da força muscular inspiratória após treinamento específico destes múscu$\operatorname{los}^{14-22}$. Condessa ${ }^{23}$ realizou uma pesquisa com dez pacientes traqueostomizados submetidos ao treinamento dos músculos respiratórios com um resistor de carga linear, realizando-se três a cinco séries de seis repetições, uma vez ao dia, por cinco a sete dias na semana. Verificou-se que somente um dos pacientes que foi submetido ao treinamento teve insucesso no desmame.

Sprague $^{24}$ utilizou o treinamento muscular com o ThresholdIMT em seis pacientes pós-cirúrgicos submetidos à VM, com tempo médio de internação de 72 dias e diagnosticados como "falhos para o desmame". O protocolo foi realizado uma vez ao dia, por seis a sete dias na semana, utilizando-se quatro séries de seis repetições, com descanso de cinco a dez minutos entre as séries. Obteve sucesso no desmame com um tempo médio de VM de dezessete dias, nos quais a PImáx aumentou de 22,5 para $54 \mathrm{~cm} / \mathrm{H}_{2} \mathrm{O}$.
Uma pesquisa recente ${ }^{25}$ realizou TMR com o Threshold IMT em 92 pacientes em desmame da VM. Os indivíduos foram divididos em Grupo Controle e em Grupo Experimental. O Grupo Experimental realizou o treinamento com uma carga de $40 \%$ de sua PImáx, com um regime de cinco séries de dez respirações, duas vezes por dia, sete dias por semana. Concluiu-se que o treinamento não encurtou significativamente o tempo de desmame, mas aumentou as PEmáx e o VC.

A FR aumentou significativamente no GI e reduziu clinicamente no GII, concordando com Bianchi et al. ${ }^{17}$, que afirma que os efeitos do TMR sobre a ventilação pulmonar estão associados com diminuição da FR, em decorrência do aumento da profundidade da respiração.

Observou-se também que o GII apresentou valores semelhantes de VC durante o desmame. A transferência do trabalho do ventilador ao paciente exige que este último seja capaz de gerar volume adequado às necessidades ventilatórias e isto depende também da força muscular, sendo que a capacidade de gerar volume diminui as complicações, especialmente as pulmonares ${ }^{26,27}$.

É importante ressaltar que não houve necessidade de interrupção do treinamento em nenhum dos casos, já que a PAS não ultrapassou os valores de normalidade e a saturação de oxigênio apresentou aumento favorável, mostrando os benefícios do treinamento em relação à oxigenação arterial, além da segurança de poder ser utilizado com pacientes críticos e com patologias neurológicas.

A limitação deste estudo foi o pequeno tamanho da amostra, que não permite extrapolar os resultados para a população de pacientes em processo de desmame da VM, bem como o número reduzido de sessões e repetições do treinamento.

Com base nestes resultados, percebe-se que o TMR instituído neste estudo foi benéfico na manutenção da força muscular respiratória, VC, FR e FC. Conclui-se que o TMR com o uso do Threshold $I M T^{\varpi}$ pode ser um aliado da fisioterapia respiratória intensiva, podendo auxiliar no processo de desmame.

\section{REFERÊNCIAS}

1. Choi J, Tasota FJ, Hoffman LA. Mobility interventions to improve outcomes in patients undergoing prolonged mechanical ventilation: a review of the literature. Biol Res Nurs. 2008;10(1):21-33. http://dx.doi.org/10.1177/1099800408319055

2. Faria ICB, Abate AK, Rezende IMO, Silva IMM, Ávila TRO. Avaliação da capacidade inspiratória em crianças com distrofia muscular progressiva. Fisioter Mov. 2008;21(1):57-63.

3. Petrof BJ, Jaber S, Matecki S. Ventilator-induced diaphragmatic dysfunction. Curr Opin Crit Care. 2010;16(1):19-25. http://dx.doi.org/10.1097/MCC.0b013e328334b166

4. Associação de Medicina Intensiva Brasileira [Internet]. Retirada (desmame) da ventilação mecânica. Disponível em: http://www. sbp.com.br/pdfs/Retirada_da_VM.pdf. Acesso em: 28 set. 2012.
5. Souza E, Terra ELSV, Pereira R, Chycaiban L, Silva J, SampaioJorge F. Análise eletromiográfica do treinamento muscular inspiratório sob diferentes cargas do Threshold IMT. Perspect Online. 2008;2(7):103-12.

6. Chiappa GRS. Efeitos do Treinamento Muscular Inspiratório em pacientes com Insuficiência Cardíaca: impacto na capacidade funcional, na oscilação da ventilação, e na qualidade de vida. Dissertação (Mestrado) - Universidade Federal do Rio Grande do Sul, Porto Alegre; 2003.

7. Cader S, Silva EB, Vale R, Bacelar S, Monteiro MD, Dantas E. Efeito do treino dos músculos inspiratórios sobre a pressão inspiratória máxima e a autonomia funcional de idosos asilados. Motricidade. 2007;3(1):279-88.

http://dx.doi.org/10.6063/motricidade.3(1).682 
8. Sarmento GJ. III Consenso Brasileiro de Ventilação Mecânica Fisioterapia no paciente sob ventilação mecânica. J Bras Pneumol. 2007;33(Supl 2):S142-S50.

9. Black LF, Hyatt RE. Maximal respiratory pressures: normal values and relationship to age and sex. Am Rev Respir Dis. 1969;99(5):696-702

10. Coelho LM, Contato C. Análise das pressões respiratórias e volumes pulmonares em pacientes traqueostomizados através do método de oclusão simples em diferentes pressões do cuf. ASSOBRAFIR Ciência. 2011;2(1):9-18.

11. Neder JA, Andreoni S, Lerario MC, Nery LE. Reference values for lung function tests. II. Maximal respiratory pressures and voluntary ventilation. Braz J Med Biol Res. 1999;32(6):719-27. http://dx.doi.org/10.1590/S0100-879X1999000600007

12. Vassilakopoulos T, Petrof BJ. Ventilator-induced diaphragmatic dysfunction. Am J Respir Crit Care Med. 2004;169(3):336-41. http://dx.doi.org/10.1164/rccm.200304-489CP

13. Chang AT, Boots RJ, Brown MG, Paratz J, Hodges PW. Reduced inspiratory muscle endurance following successful weaning from prolonged mechanical ventilation. Chest. 2005;128(2):553-9. http://dx.doi.org/10.1378/chest.128.2.553

14. Weiner P, Magadle R, Beckerman M, Weiner M, Berar-Yanay N. Maintenance of inspiratory muscle training in COPD patients: one year follow-up. Eur Respir J. 2004;23(1):61-5. http://dx.doi.org/10.1183/09031936.03.00059503

15. Pelizzaro CO, Dileoni C, Gazzana MB. Influência da fisioterapia na pressão inspiratória máxima e no tempo de ventilação mecânica invasiva. Anais do III Congresso Sul Brasileiro de Fisioterapia Respiratória: Curitiba; 2005

16. Kunikoshita LN, Silva YP, Silva TLP, Costa D, Jamami M. Efeitos de três programas de fisioterapia respiratória (PFR) em portadores de DPOC. Rev Bras Fisioter. 2006;10(4):449-55. http://dx.doi.org/10.1590/S1413-35552006000400014

17. Bianchi PDA, Valle PHC, Baldissera V, Rosa PV [Internet]. Avaliação do treinamento de força e resistência muscular respiratória com manovacuômetro e com carga linear pressórica. Disponível em: http://www.efdeportes.com/efd136/avaliacao-do-treinamentocom-manovacuometro.htm. Acesso em: 5 out. 2012.

18. Cader SA, Vale RG, Castro JC, Bacelar SC, Biehl C, Gomes MC, et al. Inspiratory muscle training improves maximal inspiratory pressure and may assist weaning in older intubated patients: a randomised trial. J Physiother. 2010;56(3):171-7. http://dx.doi.org/10.1016/S1836-9553(10)70022-9

19. Trevisan ME, Porto AS, Pinheiro TM. Influência do treinamento da musculatura respiratória e de membros inferiores no desempenho funcional de indivíduos com DPOC. Fisioter Pesq. 2010;17(3):209-13.

20. Fonseca NT, Contato C. Análise da mecânica respiratória antes e após o uso do threshold em indivíduos idosos. Rev Mineira Ciências Saúde. 2010;(2):101-8

21. Moodie LH, Reeve JC, Vermeulen N, Elkins MR. Inspiratory muscle training to facilitate weaning from mechanical ventilation: protocol for a systematic review. BMC Res Notes. 2011;4:283. http://dx.doi.org/10.1186/1756-0500-4-283

22. Barbalho-Moulim MC, Miguel GP, Forti EM, Campos FA, Costa D Effects of preoperative inspiratory muscle training in obese women undergoing open bariatric surgery: respiratory muscle strength lung volumes, and diaphragmatic excursion. Clinics (Sao Paulo). 2011;66(10):1721-7

http://dx.doi.org/10.1590/S1807-59322011001000009

23. Condessa RL. Avaliação do treinamento muscular inspiratório por threshold IMT no processo de aceleração do desmame da ventilação mecânica. Dissertação (Mestrado) - Universidade Federal do Rio Grande do Sul, Porto Alegre; 2008.

24. Sprague SS, Hopkins PD. Use of inspiratory strength training to wean six patients who were ventilator-dependent. Phys Ther. 2003; 83(2):171-81.

25. Condessa RL, Brauner JS, Saul AL, Baptista M, Silva AC, Vieira SR Inspiratory muscle training did not accelerate weaning from mechanica ventilation but did improve tidal volume and maximal respiratory pressures: a randomised trial. J Physiother. 2013;59(2):101-7. http://dx.doi.org/10.1016/S1836-9553(13)70162-0

26. Hulzebos EH, Helders PJ, Favié NJ, De Bie RA, Brutel de la Riviere A, Van Meeteren NL. Preoperative intensive inspiratory muscle training to prevent postoperative pulmonary complications in highrisk patients undergoing CABG surgery: a randomized clinical trial. JAMA. 2006;296(15):1851-7. http://dx.doi.org/10.1001/jama.296.15.1851

27. Stein R, Maia CP, Silveira AD, Chiappa GR, Myers J, Ribeiro JP. Inspiratory muscle strength as a determinant of functional capacity early after coronary artery bypass graft surgery. Arch Phys Med Rehabil. 2009;90(10):1685-91.

http://dx.doi.org/10.1016/j.apmr.2009.05.010 